\title{
An useful complication of emergent pericardiocentesis procedure in cardiac tamponade: Case report
}

\author{
Çiğdem İleri' ${ }^{1}$ Ahmet Altuğ Çinçin¹, Zekeriya Doğan¹, Bülent Mutlu²
}

\footnotetext{
1) Marmara University Faculty of Medicine, Department of Cardiology, MD, Istanbul, Turkey.

2) Marmara University Faculty of Medicine, Department of Cardiology, Prof. Dr., Istanbul, Turkey
}

\begin{abstract}
We present a case of 19 years old patient whom was admitted to emergency service with abdominal pain and dyspnea and had prodromal symptoms for about ten days. He was hypotensive, tachycardic and dyspneic. CT scan showed large amount of free peritoneal, pericardial and bilateral pleural fluid. With the diagnosis of cardiac tamponade; emergent pericardiocentesis was done with apical approach and quick hemodynamic relief was observed. It was understood that rapid haemodynamic response was a result of complication, characterized by increasing of left pleural effusion.
\end{abstract}

Key Words: Complication, abdominal pain, cardiac tamponade, pleural effusion.

İleri Ç., Çinçin A. A., Doğan Z., Mutlu B. An useful complication of emergent pericardiocentesis procedure in cardiac tamponade: Case report. EJCM 2018; 06 (1): 15-18. Doi: Doi: 10.15511/ejcm.18.00115. 


\section{Case Report}

A 19 years old man with a history of migraine and smoking, was admitted to emergency service with abdominal pain, nausea, dyspnea and loss of appetite for one week. The abdominal pain was worsening by standing and relieving by lying down. He had prodromal symptoms for ten days. Patient was consulted to our department due to detection of minimal cardiac silhouette enlargement in chest X-ray and sinus tachycardia in electrocardiogram. Computerized tomographic (CT) scan of abdomen and thorax revealed large amount of free peritoneal, $30 \mathrm{~mm}$ of pericardial, $30 \mathrm{~mm}$ of pleural effusion in left lung as well as $20 \mathrm{~mm}$ of effusion in right lung. Transthoracic echocardiography (TTE) showed large circumferential pericardial effusion (PE) with right atrial (RA) systolic and right ventricular (RV) diastolic collapse and $>30 \%$ variation in mitral inflow pattern suggesting cardiac tamponade (Figure 1).

Hypotension, tachycardia and pulsus paradoxus was observed at physical examination. After echocardiographic examination, it was concluded that the most convenient way for pericardiocentesis (PC) was apical approach. However collecting very small amount of pericardial fluid $(15 \mathrm{cc})$, his hemodynamic profile was rapidly improved. A thoracal CT scan was taken immediately, although $15 \mathrm{~mm}$ residual pericardial effusion was implemented; $20 \mathrm{~mm}$ effusion at right and $45 \mathrm{~mm}$ at left lung was identified (Figure 2).

Thoracal drainage tube was inserted left pleural cavity and $500 \mathrm{cc}$ of hemorrhagic pleural fluid was collected. Tube was removed 2 days after, and no residual or recurrent effusion existed in the control imaging. Increase in acute phase reactants like, CRP:93 (0$5 \mathrm{mg} / \mathrm{l})$ fibrinogen:744 (200-400mg/dl), ESR: $53 \mathrm{~mm} / \mathrm{h}$ and neutrophilic leucocytosis was established in blood tests. However pericardial effusion was exudative quality; aerob- anaerob blood, urine and fluid culture was negative and no acid fast bacilli was seen in fluid; ANA, Anti-CCP, Anti-DNA and ENA profile were also normal. Colchicum $2 * 5 \mathrm{mg}$ and brufen $3 * 600 \mathrm{mg}$ without antibiotic regimen applied as the treatment strategy. 10 days after beginning of the treatment, only $10 \mathrm{~mm}$ pericardial effusion at posterior wall and $8 \mathrm{~mm}$ at RV neighbouring was found in TTE (Figure 3). He was on treatment for two months without any symptoms; rheumatologic and genetic tests are still having been done.

\section{Discussion}

Cardiac tamponade is the hemodynamic result of increase in pericardial fluid which elevates intrapericardial pressure and prevent filling. ${ }^{1}$ Pulsus paradoxus, tachycardia, elevated jugular venous pressure and hypotension are non-specific but important clues for the diagnosis. Echocardiography is an important tool to assess existence of effusion and also its hemodynamic results. $^{2}$

Cardiac tamponade is critical condition and requires emergent treatment. ${ }^{1}$ A variety of percutaneous or surgical therapeutic methods have been applied. Pericardiocentesis (PC), pleuro-pericardial window, subxiphoid pericardial drainage and pericardioscopy are the treatment option. ${ }^{3}$ Percutaneous balloon pericardiotomy is another technique to create a nonsurgical pericardial
Figure 1. Transthoracic echocardiographic images of massive pericardial effusion and diastolic collapse of right ventricular free wall which is a sign of cardiac tamponade.

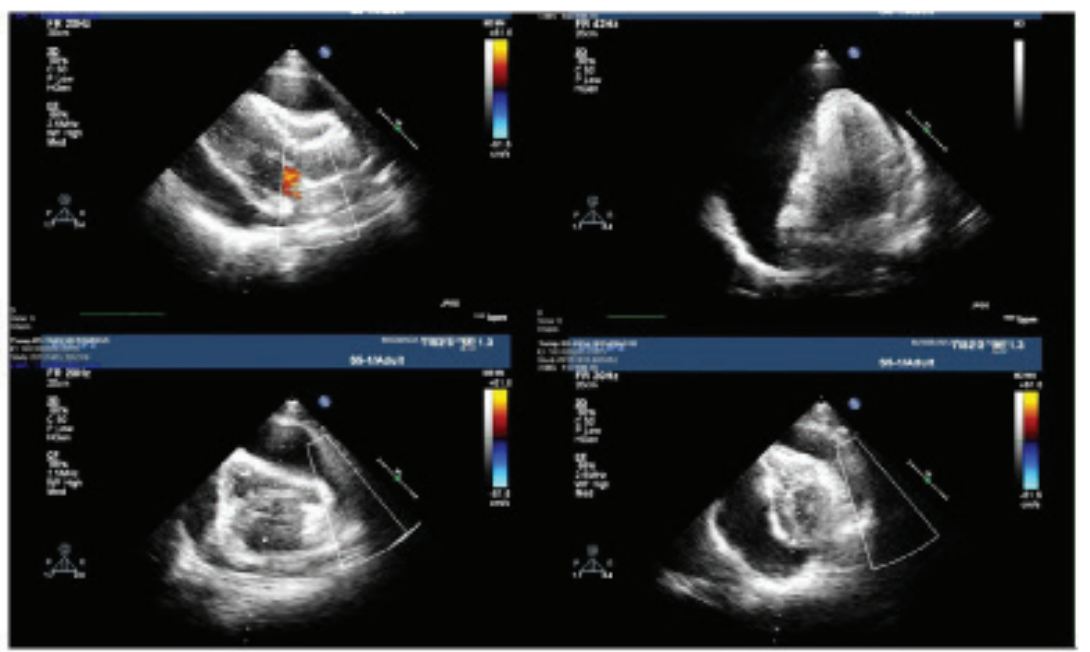


window for large pericardial effusions of critically ill patients especially with limited life expectancy. ${ }^{4,5}$

Percutaneous PC had been done blind or, electrocardiographically or fluoroscopically guided before the 2D-echocardiography-guided era. ${ }^{6,7}$ Echocardiography guided pericardiocentesis is safe and effective way for drainage of large, symptomatic pericardial effusions or in case of cardiac tamponade. ${ }^{6,8,9}$ Echocardiographic guidance facilitates detection of the puncture site, such as near the cardiac apex or sternal border or where the largest fluid collects. In a large study which investigate more than 1100 echocardiography-guided PC cases, the para-apical location was the most frequently utilized location (68\%), while the subcostal or subxiphoid locations were used only in $15 \%$ of cases .

Large observational studies of echocardiographyguided pericardiocentesis report major and minor complication rates of $<2 \%$ for non-emergent procedures. ${ }^{10,11}$ The major complications of pericardiocentesis are myocardial rupture, vascular injury, pericardial hemorrhage, air embolism, injury to the liver or abdominal viscera, infection and arrhythmia. ${ }^{12}$

In our clinic, most of the $\mathrm{PC}$ are done by apical approach with lower complication rates. In this case, a complication happened during emergent $\mathrm{PC}$ which is
Figure 2. Tomographic images of increase in amount of left pleural effusion before (left side images) and after (right side images) the pericardiocentesis procedure

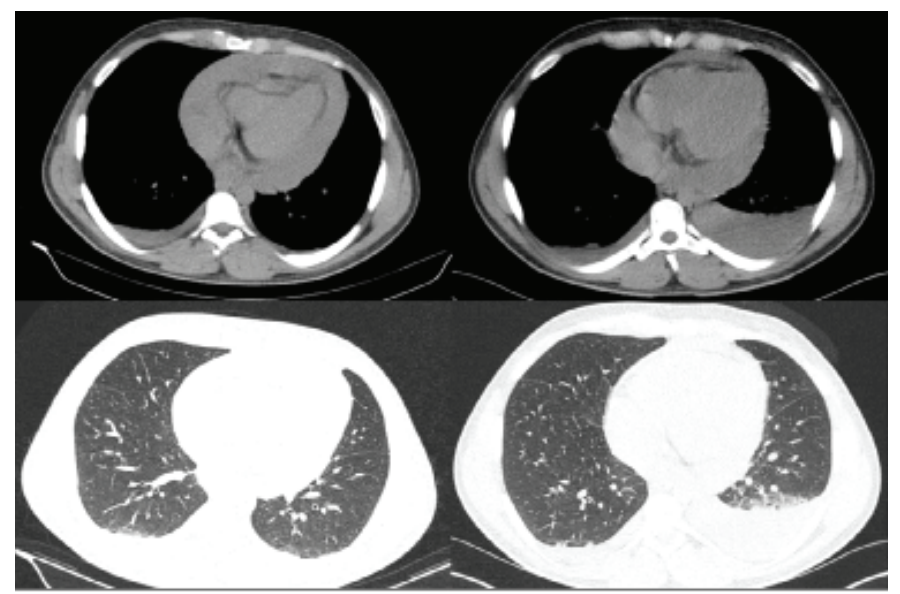

likely to occur. Despite symptomatic and haemodynamic relief of patient, immediate echocardiographic study and CT scan was taken due to continuing of clinical suspicion. Thoracal drainage tube inserted fastly as a result of increase in left pleural effusion. We think that increase in the amount of pleural fluid is an acceptable result confronting with the life threatening feature of tamponade. Mutual result of tamponade, haemodynamic parameters, clinical backround of patient as well as collaborative assesment of imaging techniques are contributing to the outcome.

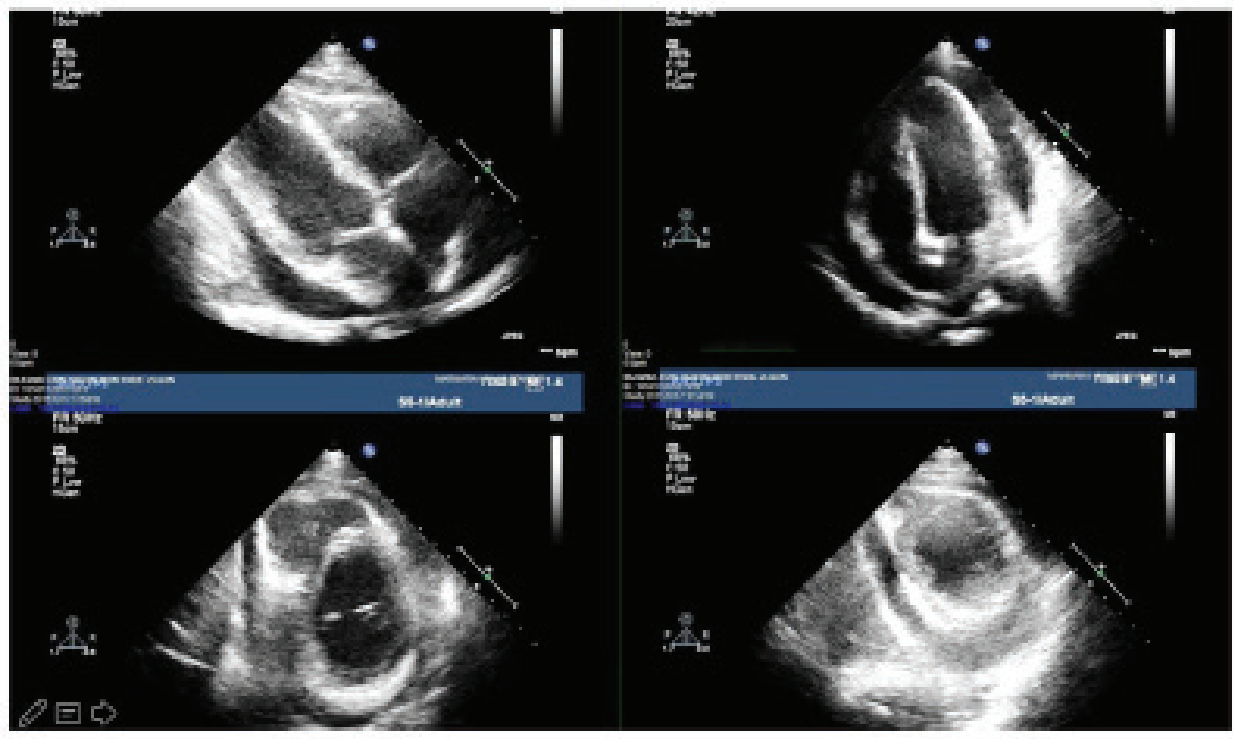

Figure 3. Transthoracic echocardiographic images of residual pericardial effusion and absence of right ventricular diastolic collapse. report. EJCM 2018; 06 (1): 15-18. Doi: Doi: 10.15511/ejcm.18.00115. 


\section{References}

1. Role of echocardiography in the treatment of cardiac tamponade. Chandraratna PA, Mohar DS, Sidarous PF. Echocardiography. 2014 Aug;31(7):899-910. doi: 10.1111/echo.12605. Epub 2014 Apr 4. Review

2. Diagnostic value of echocardiography in cardiac tamponade. Tsang TS, Oh JK, Seward JB, Tajik AJ. Herz. 2000 Dec;25(8):734-40. Review.

3. Which treatment in pericardial effusion? Campione A, Cacchiarelli M, Ghiribelli C, Caloni V, D’Agata A, Gotti G. J Cardiovasc Surg (Torino). 2002 Oct;43(5):735-9.

4. Percutaneous balloon pericardiotomy for the treatment of cardiac tamponade and large pericardial effusions: description of technique and report of the first 50 cases. Ziskind AA, Pearce AC, Lemmon CC, Burstein S, Gimple LW, Herrmann HC, McKay R, Block PC, Waldman H, Palacios IF. J Am Coll Cardiol. 1993 Jan;21(1):1-5.

5. Percutaneous balloon pericardiotomy for the treatment of infected pericardial effusion with tamponade. Aqel R, Mehta D, Zoghbi GJ. J Invasive Cardiol. 2006 Jul;18(7):E194-7. Review.

6. Clinical and echocardiographic characteristics of pericardial effusion in patients who underwent echocardiographically guided pericardiocentesis: Yonsei Cardiovascular Center experience, 1993-2003. Cho BC, Kang SM, Kim DH, Ko YG, Choi D, Ha JW, Rim SJ, Jang Y, Chung N,
Shim WH, Cho SY, Kim SS. Yonsei Med J. 2004 Jun 30;45(3):462-8.

7. Echocardiographically guided pericardiocentesis: evolution and stateof-the-art technique. Tsang TS, Freeman WK, Sinak LJ, Seward JB. Mayo Clin Proc. 1998 Jul;73(7):647-52. Review.

8. Echocardiographically guided pericardiocentesis - the gold standard for the management of pericardial effusion and cardiac tamponade. Salem K, Mulji A, Lonn E. Can J Cardiol. 1999 Nov;15(11):1251-5.

9. [Contrast medium echocardiography-assisted pericardial drainage]. Caspari G, Bartel T, Möhlenkamp S, Bersch B, von Birgelen C, Krapp J, Erbel R. Herz. 2000 Dec;25(8):755-60. German.

10. Tsang TS, Enriquez-Sarano M, Freeman WK, et al. Consecutive 1127 therapeutic echocardiographically guided pericardiocenteses: clinical profile, practice patterns, and outcomes spanning 21 years. Mayo Clin Proc 2002; 77:429.

11. Cho BC, Kang SM, Kim DH, et al. Clinical and echocardiographic characteristics of pericardial effusion in patients who underwent echocardiographically guided pericardiocentesis: Yonsei Cardiovascular Center experience, 1993-2003. Yonsei Med J 2004; 45:462.

12. Pericardiocentesis. Willner DA, Grossman SA. StatPearls [Internet] Treasure Island (FL): StatPearls Publishing; 2017 Jun-.2017 Nov 7.
Received: 11/08/2016

Accepted: 18/12/2017

Published: $15 / 03 / 2018$

Disclosure and conflicts of interest:

The authors declare no conflict of interest.

Corresponding author:

Çiğdem İleri

Mail: cgdmileri@gmail.com

İleri Ç., Çinçin A. A., Doğan Z., Mutlu B. An useful complication of emergent pericardiocentesis procedure in cardiac tamponade: Case report. EJCM 2018; 06 (1): 15-18. Doi: Doi: 10.15511/ejcm.18.00115. 